\title{
QTL mapping of agronomic traits using an RIL population derived from a cross between temperate japonica cultivars in rice (Oryza sativa L.)
}

\author{
Soo-Jin Kwon ${ }^{\dagger 1,2)}$, Young-Chan Cho ${ }^{\dagger 1)}$, Soon-Wook Kwon ${ }^{1)}$, Chang-Sik Oh ${ }^{3)}$, Jung-Pil Suh ${ }^{1)}$, \\ Young-Seop Shin ${ }^{1}$, Yeon-Gyu Kim ${ }^{1)}$, Dawn Holligan ${ }^{4)}$, Susan R. Wessler ${ }^{4}$, \\ Hung-Goo Hwang') and Sang-Nag Ahn*3) \\ 1) National Institute of Crop Science, RDA, Suwon 441-100, Korea \\ 2) National Institute of Agricultural Biotechnology, RDA, Suwon 441-100, Korea \\ 3) Department of Agronomy, Chungnam National University, Daejeon 305-333, Korea \\ 4) Department of Plant Biology, University of Georgia, Athens, GA 30602, USA
}

\begin{abstract}
To employ molecular marker-aided selection for developing japonica rice (Oryza sativa L.) cultivars with high yielding potential and good eating quality, we carried out QTL analysis for agronomic and grain traits using 231 recombinant inbred lines (RIL) from a cross between two temperate japonica cultivars, Suweon 365 and Chucheongbyeo. A linkage map with 221 loci, including 134 simple sequence repeats (SSRs), 66 amplified fragment length polymorphisms (AFLPs), and 21 miniature inverted repeat transposable elements (MITEs)-anchored markers, was constructed covering 2,227 cM of the twelve rice chromosomes, with an average distance of $11.0 \mathrm{cM}$ between markers. The proportion of polymorphic bands between parents averaged $19.5 \%, 12.9 \%$ and $42.3 \%$ for SSRs, AFLPs and MITEs, respectively. Construction of maps without AFLP or MITE markers indicated that expansion of this map is due to the incorporation of AFLP markers, and this result is consistent with previous reports. Twenty-seven QTLs for ten traits were identified over two years, and fourteen were consistently detected in two consecutive years. When the locations of each QTL detected in this study were compared with those in previous QTLs studies, eight QTLs for culm length, spikelets per panicle and, thickness, length and shape of grain, had not been reported in previous mapping studies, indicating potentially novel alleles of japonica rice. Interestingly, a cluster of seven QTLs, including heading date and grain morphology traits, was detected on the long arm of chromosome 7. The markers linked to these QTLs would be valuable in a marker-assisted breeding program for japonica cultivars.
\end{abstract}

Key Words: rice, temperate japonica, QTL, MITEs, molecular map.

\section{Introduction}

Molecular maps are available for various cross combinations in rice, Oryza sativa L. (www.gramene.org), and these maps serve several purposes, including quantitative trait loci (QTL) analysis, marker-aided selection, gene isolation, and evolutionary studies. Of the several marker types, restriction fragment length polymorphism (RFLPs) and simple sequence repeats (SSRs) have been predominantly employed in the construction of molecular maps in rice (Oryza sativa L.). Amplified fragment length polymorphism (AFLP) analysis was also used to complement other marker systems because AFLP markers might be derived from any portion of the genome that can be digested with restriction enzymes (Vos et al. 1995).

Miniature inverted repeat transposable elements

Communicated by M. Yano

Received December 13, 2007. Accepted June 6, 2008.

*Corresponding author (e-mail: ahnsn@cnu.ac.kr)

$\dagger$ These auhtors contributed equally to this work
(MITEs) are currently being developed in several crop species (Wessler 2006). They display a very high copy number of repetitive sequences and even distribution in the rice genome. This feature would facilitate mapping in rice because of the ability to generate a large number of restriction fragments in a single reaction, thus increasing the possibility of identifying polymorphisms and expediting the construction of linkage maps (Casa et al. 2000, Wessler 2006).

QTL analysis has been greatly facilitated by the development of molecular markers. Using molecular maps combined with QTL mapping technology, it is possible to characterize and isolate loci controlling quantitative traits, such as heading date (Yano et al. 2000), plant panicle trait (Yamagishi et al. 2002), yield components (Xiao et al. 1996), and grain characters (Redoña and Mackill 1998).

So far, most QTLs have been detected using intersubspecific and inter-specific crosses (Aluko et al. 2004, Tian et al. 2006). This is due to the fact that wide crosses possessed a higher level of DNA polymorphism than intrasubspecific crosses and thereby contributed to increase mapping efficiency (Causse et al. 1994); however, this 
presented a disadvantage for determining the utility of a QTL for a breeding program that exclusively utilized a japonica germplasm. Therefore, the necessity of QTL identification based on the variation of crosses between two related varieties belonging to the same subspecies, especially the japonica/japonica cross, has been emphasized using molecular linkage maps based on japonica crosses (Fujino et al. 2004, Li et al. 2005, Tabata et al. 2007, Wada et al. 2006).

The objective of the present study was to identify QTLs for traits related to yield components and grain morphology using an RIL population derived from a cross between two temperate japonica cultivars in rice.

\section{Materials and Methods}

\section{Plant materials and trait evaluation}

"Suweon365" was crossed as a female parent with "Chucheongbyeo" in 1992. The temperate japonica rice Suweon365, derived from a cross between "Seonambyeo" and "Iri353", is a semi-dwarf, high-yielding and has medium eating quality (National Institute of Crop Science: NICS 1988). Chucheongbyeo or "Akibare" in Japanese (developed from a cross between "Mandainishiki" as the female parent and an $\mathrm{F}_{5}$ line from a Wakaba/Kinmaze cross as the male parent) was introduced from Japan in 1969. It is late maturing, tall and has good eating quality (RDA 1975). $F_{1}$ plants were selfed to produce $\mathrm{F}_{2}$ seeds and $231 \mathrm{~F}_{8}$ lines were developed from the resultant $F_{2}$ plants by the single-seed descent method. The $\mathrm{F}_{8}$ recombinant inbred line (RIL) population with the parents were field evaluated in an experimental plot at the National Institute of Crop Science (NICS), Suwon, using a completely randomized block design with two replications for two years (1999 and 2000). Ten traits were measured in the 231 RILs and the parents: days to heading (DTH), culm length (CL), panicle length (PL), panicles per plant (PN), spikelets per panicle (SPP), grain yield (GY), grain thickness (GT), grain length (GL), grain width (GW) and grain length/width ratio (GS). Trait evaluation followed the procedures described in Yoon et al. (2006).

For all traits, 10 plants per each line in both years were measured and the average measurements were used for QTL analysis. Grain yield (GY) was evaluated by averaging the grain yield of 10 plants selected from the center of each plot and dried to $10 \%$ moisture content. Grain thickness (GT), grain length (GL), grain width (GW) and grain length/width ratio (GS) were measured for 50 grains per plant.

\section{DNA extraction and molecular analysis}

DNA was extracted from fresh young leaves of the 231 RILs in bulk following the procedures described in Causse et al. (1994). AFLP analysis was performed as described by Vos et al. (1995) with minor modifications. Informative combinations from polymorphism screening between the parents were selected for genotyping the RILs. The nomenclature for AFLP markers was derived from the enzyme combination, primer combination and the relative fragment number. A typical AFLP marker, em241, which is mapped on chromosome 4, indicated that the marker was amplified with the primer combination em24 (e for EcoRI, m for MseI, 2 for the specific overhang to the EcoRI end, 4 for the specific overhang to the $M s e I$ end, and 1 for the number of the band). General procedures for the transposon display (TD) modified from the AFLP method were performed as described in Casa et al. (2000) with the following modifications. Element-specific primers were derived from the long terminal repeat (LTR) sequence of Dasheng and mPing. Touch-down polymerase chain reaction (PCR) was performed for selective amplification with the following conditions: $94^{\circ} \mathrm{C}$ for $2 \mathrm{~min}$ followed by 25 cycles of $94^{\circ} \mathrm{C}$ for 30 sec, $65^{\circ} \mathrm{C}$ for $30 \mathrm{sec}$ (with a $1^{\circ} \mathrm{C}$ decrease per cycle to $58^{\circ} \mathrm{C}$ ), and $72^{\circ} \mathrm{C}$ for $60 \mathrm{sec}$, and additional incubation at $72^{\circ} \mathrm{C}$ for 5 $\mathrm{min}$. The nomenclature for the MITE-anchored marker was derived from the transposon name and the relative fragment number. PCR products were separated on a $5 \%$ polyacrylamide gel and detected by a silver-staining procedure (Promega, USA). Polymorphic bands for each primer were numbered serially in descending order of fragment size from the top to the bottom of the gel image. All bands were treated as dominant markers. Weak bands whose signals were less than $50 \%$ of the main band were not scored.

\section{Molecular linkage map construction and QTL analysis}

The Mapmaker program was used to establish a molecular map (Lander et al. 1987). All pairs of linked markers were identified using the "group" command with an LOD value of 2.0. The "orders" and "compare" commands in Mapmaker were used to identify the most probable marker order within a linkage group. The "ripple" command was used to verify the order. Additional markers were assigned to intervals using the "try" and "ripple" commands. Map distance (cM) was expressed in Kosambi function (Kosambi 1944). Assignment of linkage groups to the respective chromosomes was based on rice maps developed by Temnykh et al. (2000). Chi-square tests were performed to examine segregation ratios at the marker loci for deviation from the expected ratio of $1: 1$, and skewing was determined.

The chromosomal locations of QTLs were determined by single-point analysis (SPA) and composite interval mapping (CIM). Primary analysis using SPA was performed using the QGene program (Nelson 1997). In SPA, a QTL was declared if the phenotype was associated with a marker locus at $P<0.001$ or with two adjacent marker loci at $P<0.05$. To identify additional QTLs and to increase the resolution of QTL locations, CIM analysis was performed using QTL Cartographer v2.0 (Basten et al. 1997). Significance thresholds for CIM were determined using 1,000 permutations for each trait. For CIM, the experiment-wise significance level of $P<0.01$ corresponded to an average LOD $>3.80$, while the level of $P<0.05$ corresponded to a LOD $>2.52$. QTLs detected by SPA or CIM were not always in agreement. The QTL reported in this study was detected by both methods. The proportion of observed phenotypic variation attributable to a 
particular QTL was estimated by the coefficient of determination $\left(R^{2}\right)$. The total phenotypic variance explained was estimated by fitting a model simultaneously, including all putative QTLs for the respective trait. The effect of digenic interaction for each trait with two-way ANOVA was analyzed at the significance level of $P<0.01$ using the SAS program.

\section{Results}

\section{Trait evaluation and correlation}

The phenotype data of the traits were similar for the two years (Table 1). Trait distributions were contiguous and showed transgressive segregation with a number of RILs higher or lower than the parent of each trait. These results indicated the involvement of several genes in the control of these traits. Means and ranges for the ten traits are summarized in Table 1. Chucheongbyeo is late maturing, tall and lower yielding with short grains than Suweon365. The mean trait values of the RILs scored similarly to the mid-parent values.

Correlation coefficients among the traits are shown in Table 2. DTH showed significant positive correlations with CL $(p<0.01)$. PN and SPP were negatively correlated. CL and GY showed the highest correlations $(r=0.368-0.485)$ among the yield components in both years. Correlation

Table 1. Variation and relationship of agronomic traits of RILs in two years, 1999 and 2000

\begin{tabular}{|c|c|c|c|c|c|c|c|c|c|}
\hline \multirow{3}{*}{ Traits } & \multicolumn{4}{|c|}{1999} & \multicolumn{4}{|c|}{2000} & \multirow{3}{*}{$\begin{array}{c}\text { Correlation } \\
\text { between years }\end{array}$} \\
\hline & \multirow{2}{*}{$\mathrm{SS}^{a}$} & \multirow{2}{*}{$\mathrm{CC}$} & \multicolumn{2}{|c|}{ RILs } & \multirow{2}{*}{$\mathrm{SS}^{a}$} & \multirow{2}{*}{$\mathrm{CC}$} & \multicolumn{2}{|c|}{ RILs } & \\
\hline & & & Mean & Range & & & Mean & Range & \\
\hline DTH (days) & 104 & 114 & $108 \pm 5.0$ & $98-120$ & 106 & 113 & $110 \pm 5.8$ & $99-126$ & $0.775 * *$ \\
\hline $\mathrm{CL}(\mathrm{cm})$ & 79.8 & 89.6 & $84 \pm 8.2$ & $61-102$ & 71.2 & 88.0 & $85 \pm 8.8$ & $60.5-103$ & $0.652 * *$ \\
\hline PL (cm) & 19.9 & 18.1 & $19.6 \pm 1.2$ & $16.0-23.2$ & 21.7 & 19.4 & $20.9 \pm 1.3$ & $16.6-24.0$ & $0.246^{* *}$ \\
\hline PN (no.) & 15 & 17 & $15.0 \pm 2.0$ & $10-22$ & 13 & 14 & $13.6 \pm 1.6$ & $10-19$ & $0.160 * *$ \\
\hline SPP (no.) & 92 & 88 & $91 \pm 14.5$ & $56-144$ & 113 & 95 & $108 \pm 14.7$ & $72-155$ & $0.400 * *$ \\
\hline GY (g) & 627 & 580 & $649 \pm 74.5$ & 385-984 & 543 & 527 & $547 \pm 68.5$ & $313-801$ & $0.450 * *$ \\
\hline GT (cm) & 2.01 & 2.06 & $2.06 \pm 0.06$ & $1.82-2.20$ & 1.98 & 2.01 & $2.00 \pm 0.08$ & $1.77-2.08$ & $0.148^{*}$ \\
\hline GL (cm) & 5.07 & 4.88 & $5.02 \pm 0.13$ & $4.72-5.38$ & 5.06 & 4.67 & $4.89 \pm 0.17$ & $4.05-5.31$ & $0347 * *$ \\
\hline $\mathrm{GW}(\mathrm{cm})$ & 2.86 & 2.79 & $2.80 \pm 0.09$ & $2.58-3.00$ & 2.85 & 2.77 & $2.78 \pm 0.08$ & $2.56-3.03$ & $0.216^{* *}$ \\
\hline GS & 1.82 & 1.75 & $1.79 \pm 0.06$ & $1.66-1.98$ & 1.77 & 1.69 & $1.76 \pm 0.07$ & $1.58-1.77$ & $0.368 * *$ \\
\hline
\end{tabular}

DTH Days to heading, CL Culm length, PL Panicle length, PN Panicles per plant, SPP Spikelets per panicle, GY Grain yield, GT Grain thickness, GL Grain length, GW Grain width, GS Grain shape in traits.

${ }^{a}$ SS Suweon365, CC Chucheongbyeo

$b * * *$ Significant at the $5 \%$ and $1 \%$ levels, respectively.

Table 2. Correlation coefficients among ten agronomic traits for two years

\begin{tabular}{|c|c|c|c|c|c|c|c|c|c|c|}
\hline Traits & Year & DTH & $\mathrm{CL}$ & PL & PN & SPP & GY & GT & GL & GW \\
\hline \multirow[t]{2}{*}{$\mathrm{CL}$} & 1999 & $0.330^{* *}$ & & & & & & & & \\
\hline & 2000 & $0.314 * *$ & & & & & & & & \\
\hline \multirow[t]{2}{*}{ PL } & 1999 & 0.108 & $0.254^{* *}$ & & & & & & & \\
\hline & 2000 & $-0.457 * *$ & $0.166^{*}$ & & & & & & & \\
\hline \multirow[t]{2}{*}{$\mathrm{PN}$} & 1999 & $0.224 * *$ & 0.101 & 0.06 & & & & & & \\
\hline & 2000 & 0.084 & 0.066 & $-0.264 * *$ & & & & & & \\
\hline \multirow[t]{2}{*}{ SPP } & 1999 & -0.104 & 0.092 & 0.112 & $-0.149^{*}$ & & & & & \\
\hline & 2000 & $-0.175^{*}$ & 0.085 & $0.402 * *$ & $-0.385 * *$ & & & & & \\
\hline \multirow[t]{2}{*}{ GY } & 1999 & $0.144^{*}$ & $0.368^{* *}$ & $0.253 * *$ & 0.074 & 0.046 & & & & \\
\hline & 2000 & 0.085 & $0.485^{* *}$ & $0.322 * *$ & 0.039 & $0.317 * *$ & & & & \\
\hline \multirow[t]{2}{*}{ GT } & 1999 & -0.087 & -0.002 & 0.042 & -0.012 & 0.039 & 0.005 & & & \\
\hline & 2000 & $0.185^{* *}$ & 0.027 & -0.042 & -0.125 & 0.046 & 0.046 & & & \\
\hline \multirow[t]{2}{*}{ GL } & 1999 & $-0.145^{*}$ & 0.114 & $0.263^{* *}$ & 0.044 & -0.088 & $0.195 * *$ & 0.077 & & \\
\hline & 2000 & $-0.259 * *$ & -0.027 & $0.272 * *$ & -0.045 & 0.088 & 0.067 & 0.02 & & \\
\hline \multirow[t]{2}{*}{ GW } & 1999 & 0.054 & -0.006 & 0.041 & -0.029 & 0.078 & 0.055 & $0.256^{* *}$ & 0.109 & \\
\hline & 2000 & $-0.347 * *$ & -0.036 & $0.249 * *$ & -0.126 & $0.169^{*}$ & 0.033 & $0.302 * *$ & $0.205^{* *}$ & \\
\hline \multirow[t]{2}{*}{ GS } & 1999 & $-0.187 * *$ & 0.051 & $0.143 *$ & 0.037 & -0.064 & 0.098 & -0.12 & $0.684 * *$ & $-0.572 * *$ \\
\hline & 2000 & 0.038 & 0.006 & 0.047 & 0.059 & -0.055 & 0.032 & $-0.205^{* *}$ & $0.666^{* *}$ & $-0.569 * *$ \\
\hline
\end{tabular}

DTH Days to heading, CL Culm length, PL Panicle length, PN Panicles per plant, SPP Spikelets per panicle, GY Grain yield, GT Grain thickness, GL Grain length, GW Grain width, GS Grain shape.

$*$,** Significant at the $5 \%$ and $1 \%$ levels, respectively. 
coefficients of DTH with other traits were different between 1999 and 2000. This might have been due to the cool temperature in early summer in 2000 which affected the heading date and other traits depending on the lines.

\section{Polymorphisms between parents}

Three different types of molecular markers, SSR, AFLP and MITE-anchored, were employed for developing the temperate japonica map. For SSR, 121 out of 621 markers detected polymorphisms between the parents (19.5\%). Forty-eight AFLP primer combinations were screened for the parental polymorphism. Of these, eleven EcoRI (+2)/ MseI (+3) combinations with different overhangs were selected to genotype the RILs. The number of bands generated by each primer combination ranged from 47 to 113 with a mean of 82.5 bands. Of the 908 AFLP bands, 118 were polymorphic (12.9\%). This level of polymorphism with SSR and AFLP was lower than that observed between the intersubspecific cross (Cho et al. 1998) and similar to that between japonica cultivars (Takeuchi et al. 2001, Tabata et al. 2007). This is attributable to the narrow genetic variation between the parents as both were japonica types. On the transposon display (TD), two MITE families were employed. The reactions generated 50-80 bands in the range of 100-700 bp, similar to the AFLP profile. MITE-TD generated 52 polymorphic bands with 14 and 38 bands by Dasheng and mPing primers, respectively. The average polymorphism ratios in MITE-TD were $28.6 \%$ and $51.4 \%$ with Dasheng and $m$ Ping, respectively, which were higher than the SSR and AFLP markers.

\section{Segregation distortion of markers}

All markers mapped on the molecular map were tested for segregation distortion with a $\chi^{2}$ test for goodness of fit. Eleven loci $(5.2 \%)$ deviated from the expected $1: 1$ segregation ratio at $P \leq 0.05$. These loci were located on chromosomes 4, 6, 9, 11 and 12. No complete non-recombinant was observed in this population. Segregation distortion has been documented in several crosses (Causse et al. 1994). The lev- el detected in the present study was lower than that reported in other mapping populations, which is due to the employment of closely related japonica cultivars as parents.

\section{Construction of the japonica linkage map}

A total of 221 SSR, AFLP and MITE-anchored markers have been mapped with the 121 SSR loci as framework markers. All marker positions were in good agreement with previous maps (Causse et al. 1994, Temnykh et al. 2000). Integration of AFLP and MITE-anchored markers into the SSR map generated a map with $2,227 \mathrm{cM}$ of rice chromosomes with an average interval size of $11.0 \mathrm{cM}$ between markers (Table 3). The genome coverage of the map per chromosome ranged from $76.8 \%$ (chromosome 8 ) to $98.2 \%$ (chromosome 11) based on the reported map (Temnykh et al. 2000, McCouch et al. 2002), and several genomic regions with a low density of markers appeared as large gaps on the map. In particular, chromosome 9 had the shortest distance with the lowest number of loci (nine SSR loci). On the other hand, chromosomes 7, 10, 11 and 12 had the highest number of loci and map distances. AFLP markers were mainly distributed on chromosomes 7, 11 and 12, leading to an expansion of these chromosomes. Clustering of AFLP markers was observed on most of the mapped chromosomes. On the other hand, 21 MITE-anchored markers were evenly distributed among and within chromosomes (Table 3 and Fig. 1).

\section{QTL mapping}

Twenty-seven significant QTLs were identified for the ten traits evaluated in 1999 and 2000 (Table 4), which fourteen were detected in both years and the other thirteen were identified in only one of the two years (Fig. 1 and Table 4). No digenic interaction of each trait with two-way ANOVA was detected at the significance level of $P<0.01$.

Days to heading: Four (dth6, dth7, dth8, and dth10) of five putative QTLs were identified in both years. The Chucheongbyeo alleles increased the number of days to heading except for the $d$ th 6 locus.

Table 3. Number of integrated markers and length per chromosome

\begin{tabular}{|c|c|c|c|c|c|c|c|c|c|c|c|c|c|c|c|}
\hline \multirow[b]{2}{*}{ Marker type } & \multicolumn{2}{|c|}{$\begin{array}{l}\text { No. amplified } \\
\text { fragments }\end{array}$} & \multirow[b]{2}{*}{ Total } & \multicolumn{12}{|c|}{ Chromosome } \\
\hline & $\begin{array}{l}\text { Poly- } \\
\text { morphic } \\
(\%)\end{array}$ & $\begin{array}{l}\text { Mono- } \\
\text { morphic }\end{array}$ & & 1 & 2 & 3 & 4 & 5 & 6 & 7 & 8 & 9 & 10 & 11 & 12 \\
\hline SSR & $134(19.5)$ & 499 & 134 & 11 & 13 & 11 & 11 & 9 & 11 & 21 & 10 & 9 & 10 & 10 & 8 \\
\hline AFLP* & $118(12.9)$ & 790 & 66 & 0 & 0 & 0 & 3 & 0 & 0 & 20 & 8 & 0 & 8 & 12 & 15 \\
\hline MITE & $52(42.3)$ & 71 & 21 & 0 & 0 & 5 & 2 & 1 & 2 & 2 & 1 & 0 & 6 & 2 & 0 \\
\hline \multicolumn{3}{|l|}{ No. of markers } & 221 & 11 & 13 & 16 & 16 & 10 & 13 & 43 & 19 & 9 & 24 & 24 & 23 \\
\hline \multicolumn{3}{|l|}{ Total length (cM) } & 2,227 & 174.5 & 171.9 & 205.6 & 202.2 & 131.2 & 173.3 & 303.8 & 185.1 & 76.7 & 137.7 & 209.4 & 255.4 \\
\hline \multicolumn{3}{|c|}{ Ave. length btn markers } & $(11.0)$ & 15.9 & 13.2 & 13.2 & 12.6 & 13.1 & 13.3 & 7.1 & 9.7 & 8.5 & 5.7 & 8.7 & 11.1 \\
\hline \multicolumn{3}{|c|}{ Genome coverage $(\%)$} & (Avg. 91.0) & 80.1 & 91.2 & 87.4 & 96.0 & 95.2 & 97.8 & 90.1 & 76.8 & 82.1 & 91.1 & 98.2 & 95.3 \\
\hline
\end{tabular}

*Selective combination for AFLP analysis: AA/CAT $=\mathrm{em} 12, \mathrm{AC} / \mathrm{CAT}=\mathrm{em} 22, \mathrm{TC} / \mathrm{CAT}=\mathrm{em} 42, \mathrm{TG} / \mathrm{CAT}=\mathrm{em} 52, \mathrm{G} / \mathrm{CAT}=\mathrm{em} 62$, $\mathrm{TA} / \mathrm{CAT}=\mathrm{em} 72, \mathrm{TT} / \mathrm{CAT}=\mathrm{em} 82, \mathrm{AA} / \mathrm{CTG}=\mathrm{em} 14, \mathrm{AC} / \mathrm{CTG}=\mathrm{em} 24, \mathrm{TT} / \mathrm{CAG}=\mathrm{em} 81, \mathrm{TT} / \mathrm{CAA}=\mathrm{em} 86$ 


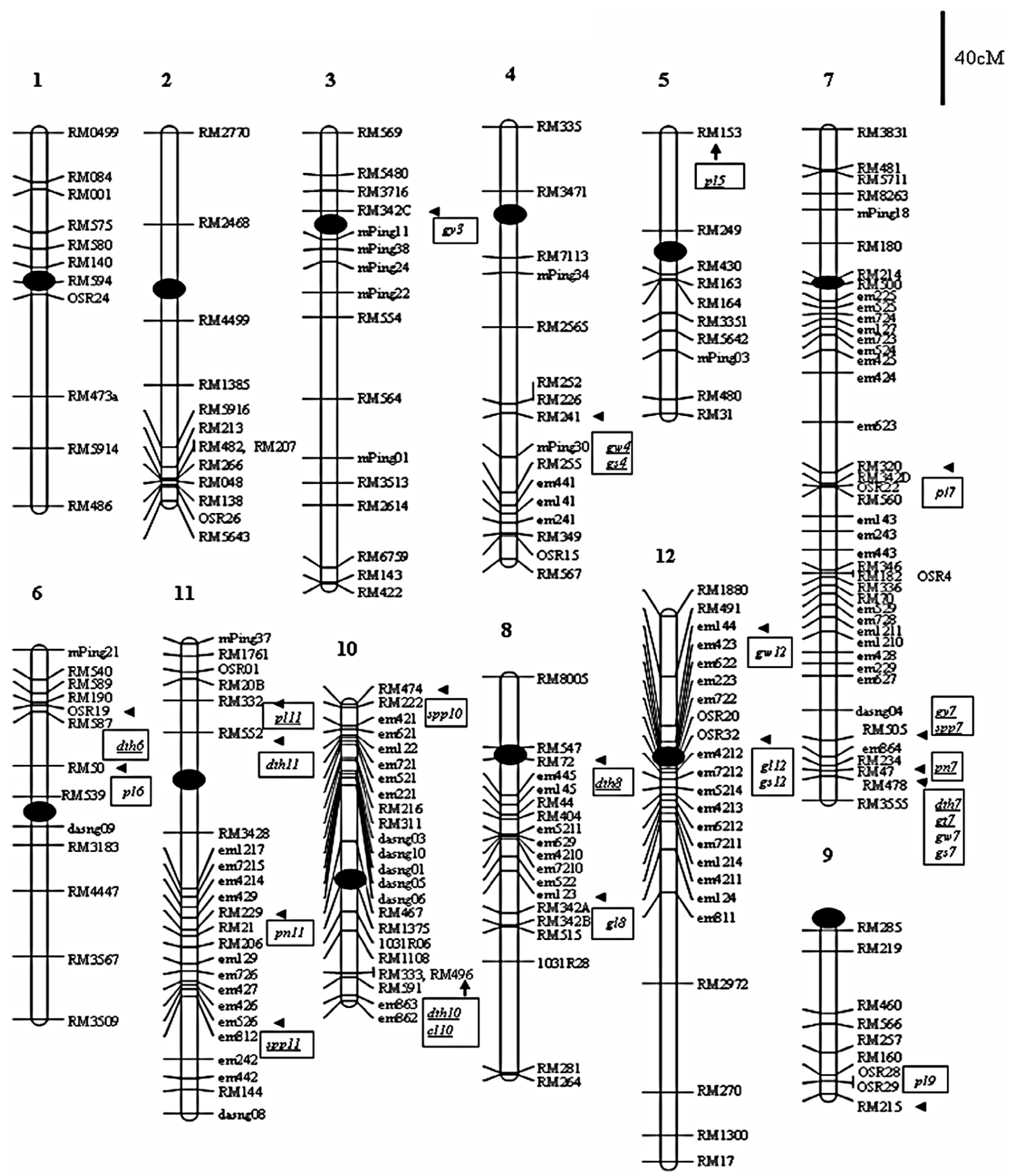

Fig. 1. The integrated map was developed using SSR, AFLP and MITE-anchored markers. Chromosome numbers are indicated above each chromosome. Designations to the right of the chromosome represent marker names and to the left represent map distances in $\mathrm{cM}$ based on the Kosambi function. QTLs detected in the same interval are boxed. QTLs detected in both years are underlined. Centromere region is indicated by black circles on the chromosome.

Culm length: One QTL (cl10) was detected in both years. This QTL explained 6.3\% of the phenotypic variance with an additive effect of $2.0 \mathrm{~cm}$ contributed by the Chucheongbyeo allele. To our knowledge, no QTL associat- ed with culm length has been reported near this chromosomal region, implying that it might be unique in japonica rice.

Panicle length: Five loci were associated with PL. pl6 and $p l 7$ shared similar genomic locations to the QTLs 
Table 4. Characteristics of putative QTLs for the ten traits detected in RILs

\begin{tabular}{|c|c|c|c|c|c|c|c|c|}
\hline Trait & QTL & CHR & $\begin{array}{l}\text { Significant } \\
\text { marker }\end{array}$ & Position $(\mathrm{cM})^{a}$ & $P$ & $\begin{array}{c}\text { Increased } \\
\text { effect }\end{array}$ & $\%$ of $\mathrm{PV}^{b}$ & $\begin{array}{l}\text { Allele } \\
\text { effect }\end{array}$ \\
\hline \multirow[t]{6}{*}{ Days to heading } & $d t h \sigma^{d}$ & 6 & OSR19 & 25.9 & 0.0000 & $\mathrm{~S}$ & 9.6 & 1.5 \\
\hline & $d t h 7^{d}$ & 7 & RM478 & 363.8 & 0.0000 & $\mathrm{C}$ & 9.1 & -2.0 \\
\hline & $d t h 8^{d}$ & 8 & RM72 & 37.8 & 0.0000 & $\mathrm{C}$ & 4.8 & -1.0 \\
\hline & $d \operatorname{th} 10^{d}$ & 10 & RM333 & 131.2 & 0.0000 & $\mathrm{C}$ & 8.1 & -1.0 \\
\hline & $d t h 11$ & 11 & RM552 & 40.0 & 0.0000 & $\mathrm{C}$ & 5.9 & -1.0 \\
\hline & & & & & & & $31.1^{e}$ & \\
\hline Culm length & $\operatorname{cll} 0^{d}$ & 10 & em863-RM333 & 131.2 & 0.0003 & $\mathrm{C}$ & 6.3 & -2.0 \\
\hline \multirow[t]{6}{*}{ Panicle length } & $p l 5^{d}$ & 5 & RM153 & 0.0 & 0.0032 & $\mathrm{~S}$ & 4.1 & 0.3 \\
\hline & pl6 & 6 & RM50 & 54.4 & 0.0020 & $\mathrm{~S}$ & 4.4 & 0.3 \\
\hline & $p l 7$ & 7 & RM320 & 224.4 & 0.0000 & $\mathrm{~S}$ & 9.1 & 0.4 \\
\hline & $p l 9$ & 9 & RM215 & 76.7 & 0.0005 & $\mathrm{C}$ & 5.3 & -0.3 \\
\hline & $p l 11^{d}$ & 11 & RM332 & 25.7 & 0.0003 & S & 5.9 & 0.3 \\
\hline & & & & & & & $21.5^{e}$ & \\
\hline \multirow[t]{3}{*}{ Panicles per plant } & $p n 7^{d}$ & 7 & RM47 & 361.3 & 0.0000 & $\mathrm{C}$ & 4.4 & -0.4 \\
\hline & pn11 & 11 & RM229 & 127.7 & 0.0080 & $\mathrm{C}$ & 3.2 & -0.4 \\
\hline & & & & & & & $5.5^{e}$ & \\
\hline \multirow[t]{4}{*}{ Spikelets per panicle } & $\operatorname{spp} 7^{d}$ & 7 & RM505 & 361.3 & 0.0043 & $\mathrm{~S}$ & 3.7 & 3.0 \\
\hline & spp10 & 10 & RM474 & 0.0 & 0.0000 & S & 7.9 & 4.0 \\
\hline & $\operatorname{spp} 11^{d}$ & 11 & em526 & 154.0 & 0.0000 & $\mathrm{~S}$ & 11.5 & 5.0 \\
\hline & & & & & & & $17.6^{e}$ & \\
\hline \multirow[t]{3}{*}{ Grain yield } & $g y 3$ & 3 & RM342C & 33.0 & 0.0174 & $\mathrm{C}$ & 2.1 & -32 \\
\hline & $g y 7^{d}$ & 7 & RM505 & 347.7 & 0.0020 & S & 2.6 & 38 \\
\hline & & & & & & & $4.1^{e}$ & \\
\hline Grain thickness & $g t 7^{d}$ & 7 & RM478 & 363.8 & 0.0000 & $\mathrm{~S}$ & 7.0 & 0.02 \\
\hline \multirow[t]{3}{*}{ Grain length } & $g l 8$ & 8 & em123 & 89.6 & 0.0003 & $\mathrm{~S}$ & 5.0 & 0.04 \\
\hline & gl12 & 12 & OSR32 & 71.5 & 0.0000 & $\mathrm{~S}$ & 7.7 & 0.04 \\
\hline & & & & & & & $9.5^{e}$ & \\
\hline \multirow[t]{4}{*}{ Grain width } & $g w 4^{d}$ & 4 & RM241 & 144.0 & 0.0000 & $\mathrm{~S}$ & 9.8 & 0.03 \\
\hline & gw7 & 7 & RM478 & 363.8 & 0.0000 & $\mathrm{~S}$ & 14.1 & 0.04 \\
\hline & $g w 12$ & 12 & em144 & 51.3 & 0.0120 & $\mathrm{C}$ & 4.5 & -0.02 \\
\hline & & & & & & & $19.5^{e}$ & \\
\hline \multirow[t]{4}{*}{ Grain length/width ratio } & $g s 4^{d}$ & 4 & RM241 & 144.0 & 0.0008 & $\mathrm{C}$ & 5.2 & -0.02 \\
\hline & gs 7 & 7 & RM478 & 363.8 & 0.0002 & $\mathrm{C}$ & 5.7 & -0.02 \\
\hline & gs 12 & 12 & OSR32 & 71.5 & 0.0000 & $\mathrm{~S}$ & 12.5 & 0.02 \\
\hline & & & & & & & $15.3^{e}$ & \\
\hline
\end{tabular}

For each QTL: The \% of PV is the percent of the phenotypic variance explained by the QTL. SS and CC refer to genotypic class defined by single markers: SS (Suweon365) or CC (Chucheongbyeo) is the homozygote, respectively. The allele effect of QTL is the difference in the phenotypic means between SS and CC classes.

${ }^{a}$ QTL position from the first marker (cM)

${ }^{b}$ Phenotypic variation explained by QTLs

${ }^{c}$ Positive value indicated the contribution from the allele of Suweon365 (SS) whereas the negative value is from Chucheogbyeo (CC)

${ }^{d}$ Putative QTL detected in both years.

${ }^{e}$ The total phenotypic variance explained simultaneously by all putative QTLs.

reported by Mei et al. (2005).

Panicles per plant: Two significant QTLs were detected on chromosomes 7 and 11, and the $p n 7$ locus was detected in both years. The Chucheongbyeo alleles contributed to the increase in panicles per plant at both loci.

Spikelets per panicle: Of the three QTLs detected, two QTLs (spp7 and spp11) were common in both years. At all loci, the Suweon 365 alleles increased spikelets per panicle.
Grain yield: Two significant QTLs, gy3 and gy7 were associated with grain yield. $g y 7$ was consistently detected in both years. The $g y 7$ locus was co-localized with QTLs for grain yield components, including days to heading, panicles per plant and spikelets per panicle, as in the other mapping studies (refer to http://www.gramene.org).

Grain thickness: One QTL, gt7, was detected in both years. The QTL gt7 explained $7.0 \%$ and $3.9 \%$ of the 
phenotypic variance in 1999 and 2000, respectively. The $g t 7$ locus was not reported in previous studies on rice.

Grain length: Two QTLs, $g l 8$ and $g l 12$ were associated with grain length (Fig. 1, Table 4). Suweon365 alleles contributed to the increase in grain length at both loci. Neither of the two QTLs was detected in the earlier QTL study (www.gramene.org).

Grain width: Three QTLs were identified for grain width and $g w 4$ was detected in both years. Although $g w 12$ was only detected in one year, this QTL explained the largest phenotypic variance (14.1\%) among the three QTLs. The Suweon 365 alleles at the $g w 4$ and $g w 7$ loci increased $\mathrm{GW}$, whereas the Chucheongbyeo allele increased GW at gw12.

Grain shape: Three QTLs, gs4, gs 7 and gs 12, affected grain shape. The gs 12 locus was co-localized with gl12 and gw12 and the other two loci shared the same region as the QTL for GT on chromosome 7.

\section{Discussion}

The linkage map, composed of 221 markers, had a map length of $2,227 \mathrm{cM}$ with an average distance of $11.0 \mathrm{cM}$ between markers (Table 3 ). This number is relatively larger than that reported in maps constructed from temperate japonica rice crosses, such as $1,060 \mathrm{cM}$ (Wada et al. 2006), and $891.1 \mathrm{cM}$ (Tabata et al. 2007). Construction of maps without AFLP or MITE markers indicated that map expansion is due to the incorporation of AFLP markers, and this result is consistent with previous reports (Ali et al. 2000, Lu et al. 1997). In this study, AFLP markers were mainly distributed on chromosomes 7,11 and 12 , leading to increased map distance in three chromosomes (approximately 260 cM). Contrary to AFLP, MITE-anchored markers did not contribute to an increase in map distance except for chromosome 3. Several genomic regions with a low density of markers appeared as large gaps on the map. Monomorphic regions are expected in the genome of a population derived from a cross between closely related temperate japonica parents. No polymorphic marker on the short arm of chromosome 9 was detected. Kobayashi et al. (2007) and Tabata et al. (2007) reported similar results for chromosome 9 in temperate japonica crosses, suggesting that chromosome 9 may be highly monomorphic and common by descent in japonica rice.

A number of QTLs affecting agronomic and grain traits have been mapped previously using populations derived from intra- or inter-subspecific and interspecific crosses (Xiao et al. 1996, Redoña and Mackill 1998, Yoon et al. 2006). Among the 27 QTLs detected in the present study, nineteen have been detected in previous QTL studies (www.gramene.org). These include the QTLs for days to heading, dth6 (Yano et al. 2000), for panicle length, pl6 and $p l 7$, in the Lemont $\times$ Teqing RI population (Mei et al. 2005), for grain yield, gy7 (Tian et al. 2006), and for grain width gw7 (Redoña and Mackill 1998). However, the other eight
QTLs, cl10 for culm length, spp10 for spikelets per panicle, $g t 7$ for grain thickness, $g l 8$ and $g l 12$ for grain length and $g s 4$, gs 7 and $g s 12$ for grain shape were identified for the first time in this study, indicating that these QTLs might be specific to temperate japonica rice. However, considering that the map showed incomplete genome coverage with gaps in several genomic regions, the number of QTLs detected in this study might be underestimated in the japonica genome. In this regard, further QTL analyses using populations derived from other parental combinations are necessary to detect more japonica-specific QTLs.

In this study, a cluster of seven QTLs was detected on the long arm of chromosome 7. Two QTLs for SPP and GY were identified near the SSR marker, RM505. The other five QTLs for DTH, PN and three grain traits, GT, GW and GS, were co-localized near SSR markers RM47 and RM478. A QTL for protein content, one of the main components for eating quality, was identified near the SSR marker RM478, using the same RIL population, and this QTL was subsequently confirmed using nearly isogenic lines segregating for the target region flanked by two SSR markers, RM234 and RM3555 (Suh et al. 2006, personal communication). In addition to this protein content QTL, this region was confirmed to harbor QTLs for DTH, GW and GT. The finding that Chucheongbyeo alleles were associated with delayed heading and low protein content is consistent with previous reports that late heading lines tend to show low protein content (Kim et al. 1994). This result seems to suggest that the co-localization of these two QTLs in this chromosomal region is due to pleiotropy, and high-resolution linkage analysis is currently underway to address this question. Although gy 7 and spp 7 need to be confirmed for their isogenic background, they are stable, considering that they were detected in both years. The availability of linked QTLs for yield, grain quality and shape traits would make it feasible to develop high-yielding japonica cultivars with good grain quality.

In this study, MITEs analysis was used to complement other marker systems because a large number of evenly distributed molecular markers can be obtained from a single reaction (Casa et al. 2000). The average polymorphism ratio (42.3\%) from Dasheng and mPing is nearly two and three times higher than that of SSR and AFLP markers, respectively. Transposable elements offer several advantages as molecular markers. MITEs are the major component of interspersed repetitive sequences and are evenly distributed in the rice genome (Casa et al. 2000, Wessler 2006). The dispersed and abundant insertion loci of many MITEs families offer a rich source of polymorphic bands in mapping populations through exploitation as PCR-based mapping tools. Furthermore, MITEs are frequently associated with genes in plants (Wessler 2006, Kwon et al. 2007). These specific characters could be exploited in the development of a powerful marker technique for the mapping and map-based cloning of genes that are related with agronomic important traits, especially in temperate japonica cultivars. At present, 221 SSR, 
AFLP, and MITE markers have been mapped in this population, providing the foundation for locating genes and QTLs. The map covers more than $91.0 \%$ of the rice genome (McCouch et al. 2002, http://www.gramene.org). The addition of recently developed SSR and MITEs markers is underway to increase the marker density and fill in the gaps.

This population offers a good foundation for the development of a japonica map for a japonica rice breeding program. As both parents are major cultivars commonly used in breeding programs, a population with marker information would be useful for marker-assisted selection and nearisogenic line development.

\section{Acknowledgements}

This study was supported in part by grants from the BioGreen21 Program (Code No. 20070301034034), RDA, and from the Technology Development Program (Code no. 306005-04-1-HD130) for Agriculture and Forestry, MAF, Republic of Korea.

\section{Literature Cited}

Ali,M.L., M.S.Pathan, J.Zhang, G.Bai, S.Sarkarung and H.T.Nguyen (2000) Mapping QTLs for root traits in a recombinant inbred population from two indica ecotypes in rice. Theor. Appl. Genet. 101: 756-766.

Aluko,G., C.Martinez, J.Tohme, C.Castano, C.Bergman and J.H.Oard (2004) QTL mapping of grain quality traits from the interspecific cross Oryza sativa $\times$ O. glaberrima. Theor. Appl. Genet. 109: 630-639.

Basten,C.J., B.S.Weir and Z.B.Zeng (1997) QTL Cartographer: a Reference Manual and Tutorial for QTL Mapping. Department of Statistics, North Carolina State University, Raleigh, NC, USA.

Casa, A.M., C.Brouwer, A.Nagel, L.Wang, Q.Zhang, S.Kresovich and S.R.Wessler (2000) The MITE family Heartbreaker (Hbr): Molecular markers in maize. Proc. Natl. Acad. Sci. USA 97: 10083-10089.

Causse,M.A., T.M.Fulton, Y.G.Cho, S.N.Ahn, J.Chunwongse, K.Wu, J.Xiao, Z.H.Yu, P.C. Ronald， S.E. Harrington， G. Second, S.R.McCouch and S.D.Tanksley (1994) Saturated molecular map of the rice genome based on an intraspecific backcross population. Genetics 138: 1251-1274.

Cho, Y.G., S.R. McCouch， M. Kuiper， M.R. Kang， J. Pot， J.T.M. Groenen and M.Y.Eun (1998) Integrated map of AFLP, SSLP and RFLP markers using a recombinant inbred population of rice (Oryza sativa L.). Theor. Appl. Genet. 97: 370-380.

Fujino,K., H.Sekiguchi, T.Sato, H.Kikuchi, Y.Nonoue, Y.Takeuchi, T.Ando, S.Y.Lin and M.Yano (2004) Mapping of quantitative trait loci controlling low-temperature germinability in rice (Oryza sativa L.). Theor. Appl. Genet. 108: 794-799.

Kim,K.H., S.Y.Cho, H.P.Moon and H.C.Choi (1994) Breeding strategy for improvement and diversification of grain quality in rice. Korean J. Breed. 26 (Supp. 2): 3-19 (in Korean).

Kobayashi,A., B.Genliang, Y.Shenghai and K.Tomita (2007) Detection of quantitative trait loci for white-back and basal-white kernels under high temperature stress in japonica rice varieties. Breed. Sci. 57: 107-116.

Kosambi,D.D. (1944) The estimation of map distance from recombi- nation values. Ann. Eugen. 12: 172-175.

Kwon, S.J.， D.H.Kim， M.H.Lim， Y.Long， J.L.Meng， K.B.Lim, J.A.Kim, J.S.Kim, M.Jin, H.I.Kim, S.N.Ahn, S.R.Wessler, T.J.Yang and B.S.Park (2007) Terminal repeat retrotransposon in miniature (TRIM) as DNA markers in Brassica relatives. Mol. Genet. Genomics 278: 361-370.

Lander,E.S., P.Green, J.Abrahamson, A.Barlow, M.J.Daly, S.E. Lincoln and L.Newbug (1987) Mapmaker: an interactive computer package for constructing primary genetic linkage maps of experimental and natural populations. Genomics 1: 174-181.

Li,Z., P.Mu, C.Li, H.Zhang, Z.Li, Y.Gao and X.Wang (2005) QTL mapping of root traits in a doubled haploid population from a cross between upland and lowland japonica rice in three environments. Theor. Appl. Genet. 110: 1244-1252.

Lu,C., L.Shen, Z.Tan, Y.Xu, P.He, Y.Chen and L.Zhu (1997) Comparative mapping of QTLs for agronomic traits of rice across environments using a doubled-haploid population. Theor. Appl. Genet. 94: 145-150.

McCouch,S.R., L.Teytelman, Y.Xu, K.B.Lobos, K.Clare, M.Walton, B.Fu, R.Maghirang, Z.Li, Y.Xing et al. (2002) Development and mapping of 2240 new SSR markers for rice (Oryza sativa L.). DNA Res. 9: 199-207.

Mei,H.W., Z.K.Li, Q.Y.Shu, L.B.Guo, Y.P.Wang, X.Q.Yu, C.S.Ying and L.J.Luo (2005) Gene actions of QTLs affecting several agronomic traits resolved in a recombinant inbred rice population and two backcross populations. Theor. Appl. Genet. 110: 649659

National Institute of Crop Science (1988) Annual report on agricultural research in rice (in Korean). p. 866.

Nelson,J.C. (1997) Qgene: software for marker-based genomic analysis and breeding. Mol. Breed. 3: 239-245.

Redoña,E.D. and D.J.Mackill (1998) Quantitative trait locus analysis for rice panicle and grain characteristics. Theor. Appl. Genet. 96: 957-963.

Rural Development Administration (1975) A handbook on cultivars of major crop (in Korean). p. 469

Suh, J.P., Y.C. Cho, S.J. Kwon, I.S. Choi， H.C. Hong, Y.G. Kim, S.N.Ahn and H.G.Hwang (2006) identification of QTLs for grain quality on RIL population derived from a cross between japonica rices. Korean J. Breed. 38: 105-112.

Tabata,M., H.Hirabayashi, Y.Takeuchi, I.Ando, Y.Iida and R.Ohsawa (2007) Mapping of quantitative trait loci for the occurrence of white-back kernels associated with high temperatures during the ripening period of rice (Oryza sativa L.). Breed. Sci. 57: $47-52$.

Takeuchi, Y., H. Hayasaka, B.Chiba, I. Tanaka, T. Shimano, M. Yamagishi, K.Nagano, T.Sasaki and M.Yano (2001) Mapping quantitative trait loci controlling cool-temperature tolerance at booting stage in temperate japonica rice. Breed. Sci. 51: 191-197.

Temnykh,S., W.D.Park, N.Ayer, S.Cartinhour, N.Hauck, L.Lipovich, Y.G.Cho, T.Ishii and S.R.McCouch (2000) Mapping and genome organization of microsatellite sequences in rice (Oryza sativa $\mathrm{L}$ ): Frequency, length variation, transposon association, and genetic marker potential. Theor. Appl. Genet. 100: 697712 .

Tian,F., Z.Zhu, B.Zhang, L.Tan, Y.Fu, X.Wang and C.Q.Sun (2006) Fine mapping of a quantitative trait locus for grain number per panicle from wild rice (Oryza rufipogon Griff.). Theor. Appl. Genet. 113: 619-629.

Vos,P., K.Hogers, M.Bleeker, M.Reijans, T.VandeLee, M.Hornes, 
B.A.Frijters, J.Pot, J.Peleman, M.Kuiper and M.Zabeau (1995) AFLP: a new technique for DNA fingerprinting. Nucleic Acids Res. 23: 4407-4414.

Wada,T., Y.Uchimura, T.Ogata, M.Tsubone and Y.Matsue (2006) Mapping of QTLs for physicochemical properties in japonica rice. Breed. Sci. 56: 253-260.

Wessler, S.R. (2006) Eukaryotic transposon element; Teaching old genome new tricks. In: Caporale, L. (ed.) The Implicit Genome, Oxford Univ. Press, USA, pp. 138-162.

Xiao,J., J.Li, L.Yuan and S.D.Tanksley (1996) Identification of QTLs affecting traits of agronomic importance in a recombinant inbred population derived from a subspecific rice cross. Theor. Appl. Genet. 92: 230-244.

Yamagishi,M., Y.Takeuchi, I.Kono and M.Yano (2002) QTL analysis for panicle characteristics in temperate japonica rice. Euphytica 128: 219-224.

Yano, M., Y. Katayose, M. Ashikari, U. Yamanouchi, L. Monna, T.Fuse, T.Baba, K.Yamamoto, Y.Umehara, Y.Nagamura and T. Sasaki (2000) $H d l$, a major photoperiod sensitivity quantitative trait locus in rice, is closely related to the Arabidopsis flowering time gene CONSTANS. Plant Cell 12: 2473-2483.

Yoon, D.B., K.H. Kang, H.J. Kim, H.G. Ju， S.J.Kwon， J.P. Suh, O.Y.Jeong and S.N.Ahn (2006) Mapping of quantitative trait loci for yield components and morphological traits in an advanced backcross population between Oryza grandiglumis and the O. sativa cultivar Hwaseongbyeo. Theor. Appl. Genet. 112: 1052-1062. 